\title{
MAPPING FLAT CRACKS ONTO PENNY-SHAPED CRACKS, WITH APPLICATION TO SOMEWHAT CIRCULAR TENSILE CRACKS
}

\author{
BY \\ P. A. MARTIN \\ Department of Mathematics, University of Manchester, U.K.
}

\begin{abstract}
Consider a three-dimensional homogeneous isotropic elastic solid containing a flat pressurized crack, $\Omega$. The problem of finding the resulting stress distribution can be reduced to a hypersingular integral equation over $\Omega$ for the crack-opening displacement. Here, this equation is transformed into a similar equation over a circular region $D$, using a conformal mapping between $\Omega$ and $D$. This new equation is then regularized analytically by using an appropriate expansion method (Fourier series in the azimuthal direction and series of orthogonal polynomials in the radial direction). Analytical results for regions that are approximately circular are also obtained. The method will generalize to other scalar problems and to vector problems (such as shear loading of the crack).
\end{abstract}

1. Introduction. Many two-dimensional problems involving thin plates or cracks can be formulated as one-dimensional hypersingular integral equations, or systems of such equations. Examples are potential flow past a rigid plate, acoustic scattering by a hard strip, and stress fields around cracks. In general, the crack or plate will be curved. For scalar problems, parametrisation of the curve leads to an equation of the form

$$
f_{-1}^{1}\left\{\frac{1}{(x-t)^{2}}+M(x, t)\right\} w(t) d t=p(x) \text { for }-1<x<1
$$

supplemented by two boundary conditions, which are often $w(-1)=w(1)=0$. Here, $w$ is the unknown function, $p$ is prescribed, and the kernel $M$ has a weaker singularity than the dominant hypersingularity. For many references and further details, see [22].

An effective way of solving (1.1), numerically, is provided by expanding $w$ using Chebyshev polynomials of the second kind, $U_{n}$ :

$$
w(t) \simeq \sqrt{1-t^{2}} \sum_{n=0}^{N} w_{n} U_{n}(t)
$$

Received March 29, 1994.

1991 Mathematics Subject Classification. Primary 31B10, 73M25. 
where $w_{0}, w_{1}, \ldots, w_{N}$ are coefficients to be found; note that this expansion incorporates the known behaviour of $w$ near the two ends of the plate [21]. The method is effective because, with this expansion, the hypersingular integral can be evaluated analytically:

$$
\oiint_{-1}^{1} \frac{\sqrt{1-t^{2}} U_{n}(t)}{(x-t)^{2}} d t=-\pi(n+1) U_{n}(x) .
$$

One can then develop a Galerkin-type method or a collocation method for determining the unknown coefficients. Such methods have been used by many authors; examples are [8], [14], and [25]. Moreover, the convergence of the collocation scheme has been proved by Golberg [10], [11] and by Ervin and Stephan [6].

Now, consider an analogous three-dimensional problem, such as potential flow past a rigid flat plate $\Omega$. We can reduce such problems to two-dimensional hypersingular integral equations over $\Omega$. In particular, if $\Omega$ is a circular disc, expansion methods can be developed: use a Fourier decomposition in the azimuthal direction together with an expansion in terms of Gegenbauer polynomials in the radial direction; again, this method is effective because it incorporates the known behaviour around the edge of $\Omega$, and it allows the two-dimensional hypersingular integral to be evaluated analytically (see Sec. 3).

What happens if $\Omega$ is not circular? In order to use an expansion method, we map $\Omega$ onto a disc $D$. However, we are not at liberty to choose any convenient mapping: typically, the mapping will modify the hypersingularity in an essential way. We must use a conformal mapping of $\Omega$ onto $D$. This preserves the structure of the hypersingularity, allowing the use of the Fourier-Gegenbauer expansion method on the transformed integral equation. This new method is described in Sec. 4.

As well as leading to a viable numerical method, the combination of conformal mapping and two-dimensional hypersingular integral equations can be used to obtain analytical results. Specifically, in Sec. 5, we consider regions $\Omega$ that are approximately circular. We obtain a rather simple result in the transform domain which is then shown to be equivalent to a result of Gao and Rice [9].

In the paper, we phrase our equations in terms of crack problems: thus, $\Omega$ represents a flat pressurized crack in a three-dimensional elastic solid. However, this problem is equivalent to potential flow past a rigid plate. Moreover, the dominant parts of the equations are also encountered in other problems, such as acoustic scattering by a soundhard plate. A discussion of further applications and developments is given in Sec. 6 .

2. Pressurized flat cracks. Consider a three-dimensional homogeneous isotropic elastic solid containing an arbitrary flat crack $\Omega$. We choose Cartesian coordinates $x, y$, and $x_{3}$, with origin $O$, so that $\Omega$ lies in the plane $x_{3}=0$ (the $x y$-plane). We assume that $\Omega$ is a simply-connected region and that $O$ is a point in $\Omega$. Let us suppose that the crack is pressurized; thus, we suppose that

$$
\tau_{33}(x, y, 0)=\frac{\mu}{1-\nu} p(x, y) \quad \text { for }(x, y) \in \Omega
$$

where $\tau_{i j}$ is the stress tensor, $\mu$ is the shear modulus, $\nu$ is Poisson's ratio, and $p(x, y)$ is (proportional to) the prescribed pressure opening the crack. 
The discontinuity in the normal component of the displacement vector across the crack, $\left[u_{3}(x, y)\right]=w(x, y)$, solves an integral equation over $\Omega$. This can be written in several equivalent forms:

$$
\begin{aligned}
\frac{1}{4 \pi}\left(\frac{\partial^{2}}{\partial x_{0}^{2}}+\frac{\partial^{2}}{\partial y_{0}^{2}}\right) \int_{\Omega} \frac{w(x, y)}{R} d \Omega & =p\left(x_{0}, y_{0}\right), \quad\left(x_{0}, y_{0}\right) \in \Omega \\
\frac{-1}{4 \pi} f_{\Omega}\left\{\frac{\partial w}{\partial x} \frac{\partial}{\partial x}\left(\frac{1}{R}\right)+\frac{\partial w}{\partial y} \frac{\partial}{\partial y}\left(\frac{1}{R}\right)\right\} d \Omega & =p\left(x_{0}, y_{0}\right), \quad\left(x_{0}, y_{0}\right) \in \Omega \\
\frac{1}{4 \pi} \mho_{\Omega} \frac{w(x, y)}{R^{3}} d \Omega & =p\left(x_{0}, y_{0}\right), \quad\left(x_{0}, y_{0}\right) \in \Omega
\end{aligned}
$$

here, $R=\left\{\left(x-x_{0}\right)^{2}+\left(y-y_{0}\right)^{2}\right\}^{1 / 2}$. These equations are to be solved subject to

$$
w(x, y)=0 \quad \text { for }(x, y) \in \partial \Omega
$$

where $\partial \Omega$ is the boundary of $\Omega$ (the crack edge); $\partial \Omega$ is a simple closed curve.

Equation (2.2) can be found in Bueckner's article [3, p. 287], where it is credited to Panasyuk [23, Eq. (VI.21)], who in turn refers to a Russian paper by M. Ya. Leonov published in 1940 .

Equation (2.3) was derived by Kossecka [16], Bui [4], and Guidera and Lardner [12]. It involves Cauchy principal-value integrals over $\Omega$, and is known as the traction BIE. For its numerical treatment, see [26] and [29].

Equation (2.4) is a hypersingular integral equation for $w$; it was first derived by Ioakimidis [13]. For its numerical treatment, see [18] and [28]; further references are given in [22]. In the sequel, we will concentrate on (2.4).

3. Penny-shaped cracks. Suppose that $\Omega$ is a penny-shaped crack of radius $a$; thus

$$
\Omega=D_{a} \equiv\{(r, \theta): 0 \leq r<a,-\pi \leq \theta<\pi\}
$$

where $r$ and $\theta$ are polar coordinates in the plane of the crack, $x=r \cos \theta, y=r \sin \theta$.

Suppose, for simplicity, that the loading is symmetric about $\theta=0$. Thus, we can write

$$
p(x, y)=\sum_{n=0}^{\infty} p_{n}(r / a) \cos n \theta .
$$

Then, the crack-opening displacement has a similar expansion,

$$
w(x, y)=a \sum_{n=0}^{\infty} w_{n}(r / a) \cos n \theta
$$

It is known that (the dimensionless functions) $w_{n}$ and $p_{n}$ are related by the formula

$$
w_{n}(r)=\frac{-4}{\pi} r^{n} \int_{r}^{1} \frac{1}{t^{2 n} \sqrt{t^{2}-r^{2}}} \int_{0}^{t} \frac{p_{n}(s) s^{n+1}}{\sqrt{t^{2}-s^{2}}} d s d t
$$


for $n=0,1,2, \ldots$; see [12]. This formula simplifies if we expand $p_{n}$ as

$$
p_{n}(r)=r^{n} \sum_{j=0}^{\infty} S_{j}^{n} \frac{C_{2 j+1}^{n+1 / 2}\left(\sqrt{1-r^{2}}\right)}{\sqrt{1-r^{2}}},
$$

where the coefficients $S_{j}^{n}$ are known and $C_{m}^{\lambda}(x)$ is a Gegenbauer polynomial of degree $m$ and index $\lambda[5, \S 10.9]$; these polynomials are orthogonal and satisfy

$$
\int_{0}^{1} \frac{r^{2 m+1}}{\sqrt{1-r^{2}}} C_{2 j+1}^{m+1 / 2}\left(\sqrt{1-r^{2}}\right) C_{2 k+1}^{m+1 / 2}\left(\sqrt{1-r^{2}}\right) d r=h_{j}^{m} \delta_{j k}
$$

where $\delta_{i j}$ is the Kronecker delta and $h_{j}^{m}$ is a known constant. It follows from (3.1) that

$$
w_{n}(r)=r^{n} \sum_{j=0}^{\infty} W_{j}^{n} C_{2 j+1}^{n+1 / 2}\left(\sqrt{1-r^{2}}\right),
$$

where the coefficients $W_{j}^{n}$ are given by

$$
W_{j}^{n}=-S_{j}^{n} \frac{(n+j) ! j !}{\Gamma(n+j+3 / 2) \Gamma(j+3 / 2)} .
$$

This result was obtained by Krenk [17]; see also [19]. It can also be expressed in terms of associated Legendre functions or in terms of Jacobi polynomials; see [20] for further references.

Similar results can be obtained for loadings that are antisymmetric about $\theta=0$. When combined with those above, we obtain the following particular result (using $C_{1}^{\lambda}(x)=$ $2 \lambda x$ ), which will be used in Sec. 5: the loading

$$
p(x, y)=-(r / a)^{n} e^{i n \theta}
$$

gives rise to the crack-opening displacement

$$
w(x, y)=\sqrt{a^{2}-r^{2}} \frac{n !}{\Gamma(n+3 / 2) \Gamma(3 / 2)}\left(\frac{r}{a}\right)^{n} e^{i n \theta} .
$$

4. Conformal mapping. Let us now consider mapping $\Omega$ onto a disc. This can be done in many ways. For example, suppose that $\Omega$ is star-shaped, so that we can choose an origin in $\Omega$ such that

$$
\Omega=\{(r, \theta): 0 \leq r<\rho(\theta),-\pi \leq \theta \leq \pi\}
$$

thus, $\partial \Omega$ is given by $r=\rho(\theta)$. Then, change variables from $r$ and $\theta$ to $s$ and $\varphi$, according to

$$
r=s \rho(\varphi), \quad \theta=\varphi
$$

this maps $\Omega$ onto the unit disc,

$$
D \equiv\{(s, \varphi): 0 \leq s<1,-\pi \leq \varphi<\pi\}
$$


If such a change of variables is made in the integral equation (2.4), we obtain a new integral equation over $D$. However, in general, this equation will have a kernel with a different singularity: instead of the kernel $R^{-3}$, the new kernel is of the form $\sigma(\Phi) S^{-3}$, where $S$ and $\Phi$ are polar coordinates at the singular point (see Appendix A for details); an integral equation with such a kernel differs essentially from the original equation for a penny-shaped crack.

The difficulty described above does not arise if we use a conformal mapping between $\Omega$ and $D$. Let $z=x+i y$ and $z_{0}=x_{0}+i y_{0}$ be complex variables. We map the region $\Omega$ in the $z$-plane onto the unit disc, $|\zeta|<1$, in the $\zeta$-plane, using the conformal mapping

$$
\zeta=F(z)
$$

The function $F$ is required to be analytic in $\Omega$. By the Riemann mapping theorem [2, p. 222], $F(z)$ exists, and is unique if we normalise it by, for example, $F(0)=0$ and $\left|F^{\prime}(0)\right|>0$.

For our purposes, the crucial property of a conformal mapping is as follows. Let $\zeta_{0}=F\left(z_{0}\right)$ and note that

$$
\left|F^{\prime}\left(z_{0}\right)\right|=\lim _{z \rightarrow z_{0}} \frac{\left|\zeta-\zeta_{0}\right|}{\left|z-z_{0}\right|}
$$

whence "... any small line segment with one end-point at $z_{0}$ is, in the limit, contracted or expanded in the ratio $\left|F^{\prime}\left(z_{0}\right)\right|$. In other words, the linear change of scale at $z_{0}$, effected by the transformation (4.2) is independent of the direction" [2, p. 74].

So, to exploit this property, we require that $\left|F^{\prime}(z)\right| \neq 0$ for $z \in \Omega$. This condition is also sufficient to ensure that we can invert (4.2). Thus, given a length-scale $a$ for $\Omega$, we have

$$
z=a f(\zeta) \text { for }|\zeta|<1
$$

as the conformal mapping of $D$ onto $\Omega$; this form is most convenient for our application. The analytic function $f$ is known to exist for any simply-connected domain $\Omega$. We assume that $\left|f^{\prime}(\zeta)\right| \neq 0$ for all $\zeta$ with $|\zeta|<1$.

First, we investigate the effect of the mapping (4.3) on the kernel of (2.4). We have

$$
R=\left|z-z_{0}\right|=a\left|f(\zeta)-f\left(\zeta_{0}\right)\right| \simeq a\left|f^{\prime}\left(\zeta_{0}\right)\right| S
$$

for small $S$, where $S=\left|\zeta-\zeta_{0}\right|$; also, by symmetry, $R \simeq a\left|f^{\prime}(\zeta)\right| S$. So, we can write

$$
a^{3} R^{-3}=\left|f^{\prime}(\zeta)\right|^{-3 / 2}\left|f^{\prime}\left(\zeta_{0}\right)\right|^{-3 / 2}\left\{S^{-3}+K\left(\zeta, \zeta_{0}\right)\right\}
$$

exactly, where the symmetric kernel $K$ is defined by

$$
K\left(\zeta, \zeta_{0}\right)=\frac{\left|f^{\prime}(\zeta)\right|^{3 / 2}\left|f^{\prime}\left(\zeta_{0}\right)\right|^{3 / 2}}{\left|f(\zeta)-f\left(\zeta_{0}\right)\right|^{3}}-\frac{1}{\left|\zeta-\zeta_{0}\right|^{3}}
$$

We anticipate that $K=O\left(S^{-2}\right)$ as $S \rightarrow 0$. However, it turns out that $K=O\left(S^{-1}\right)$ as $S \rightarrow 0$ (see Appendix B), so that $K$ only has a weak singularity. 
Next, we consider the Jacobian of the transformation: it is $a^{2}\left|f^{\prime}(\zeta)\right|^{2}$. Hence, if we put

$$
\zeta=\xi+i \eta=s e^{i \varphi} \quad \text { and } \quad \zeta_{0}=\xi_{0}+i \eta_{0}=s_{0} e^{i \varphi_{0}}
$$

we find that

$$
d \Omega=d x d y=a^{2}\left|f^{\prime}(\zeta)\right|^{2} d \xi d \eta=a^{2}\left|f^{\prime}(\zeta)\right|^{2} s d s d \varphi
$$

Finally, setting

$$
w(x(\zeta), y(\zeta))=a\left|f^{\prime}(\zeta)\right|^{-1 / 2} W(\xi, \eta) \quad \text { and } \quad p\left(x\left(\zeta_{0}\right), y\left(\zeta_{0}\right)\right)=\left|f^{\prime}\left(\zeta_{0}\right)\right|^{-3 / 2} P\left(\xi_{0}, \eta_{0}\right)
$$

we find that (2.4) becomes

$$
\frac{1}{4 \pi} \oint_{D} \frac{W(\xi, \eta)}{S^{3}} d \xi d \eta+\frac{1}{4 \pi} \int_{D} W(\xi, \eta) K\left(\zeta, \zeta_{0}\right) d \xi d \eta=P\left(\xi_{0}, \eta_{0}\right)
$$

for $\left(\xi_{0}, \eta_{0}\right) \in D$, where all quantities are dimensionless. This equation is to be solved subject to $W=0$ on $s=1$.

We can write $(4.5)$ in operator notation as

$$
(H+K) W=P
$$

The operator $K$, defined by the second term on the left-hand side of (4.5), is a weaklysingular integral operator. The hypersingular operator $H$, defined by the first term on the left-hand side of (4.5), is precisely the operator on the left-hand side of (2.4) for a penny-shaped crack. Consequently, as described in Sec. 3, we have an explicit expression for $H^{-1}$. Hence, we can write (4.6) equivalently as

$$
\left(I+H^{-1} K\right) W=H^{-1} P
$$

which is a regularized version of the hypersingular integral equation (4.5). Computationally, the following schemes suggest themselves. Expand $W$ as

$$
W(\xi, \eta)=\sum_{n=0}^{N} W_{n}(s) \cos n \varphi
$$

with

$$
W_{n}(s)=s^{n} \sum_{j=0}^{J} W_{j}^{n} C_{2 j+1}^{n+1 / 2}\left(\sqrt{1-s^{2}}\right) .
$$

Then, either use the orthogonality relation (3.2) together with the orthogonality of the trigonometric functions, leading to a Petrov-Galerkin method, or simply collocate. It is likely that a numerical analysis of these schemes can be given by extending the work of Golberg [10], [11] and of Ervin and Stephan [6] on analogous one-dimensional hypersingular integral equations. Rather than investigate these numerical questions here, we focus on analytical results for domains $\Omega$ that are approximately circular. 
5. Somewhat circular cracks. Consider the conformal mapping

$$
f(\zeta)=\zeta+\varepsilon g(\zeta)
$$

where $\varepsilon$ is a small dimensionless parameter and $g$ is analytic; we assume that

$$
g(0)=0
$$

When (5.1) is combined with (4.3), we see that the unit disc in the $\zeta$-plane is mapped into a domain $\Omega$ in the $z$-plane that is approximately a circle of radius $a$; moreover, $\zeta=0$ is mapped to $z=0$. As an example, the choice

$$
g(\zeta)=\zeta^{n+1}
$$

leads to a crack $\Omega$ with boundary $\partial \Omega$ given by

$$
r=\rho(\theta)=a(1+\varepsilon \cos n \theta),
$$

with an error of $O\left(\varepsilon^{2}\right)$; such geometries were considered by Gao and Rice [9] in their study of crack stability.

Returning to the general case, substitute (5.1) into (4.4) to give

$$
K\left(\zeta, \zeta_{0}\right)=\varepsilon K_{0}\left(\zeta, \zeta_{0}\right)+O\left(\varepsilon^{2}\right)
$$

as $\varepsilon \rightarrow 0$, where the symmetric kernel $K_{0}$ is defined by

$$
K_{0}\left(\zeta, \zeta_{0}\right)=\frac{3}{S^{3}} \operatorname{Re}\left\{\frac{1}{2}\left(g^{\prime}(\zeta)+g^{\prime}\left(\zeta_{0}\right)\right)-\frac{g(\zeta)-g\left(\zeta_{0}\right)}{\zeta-\zeta_{0}}\right\}
$$

This is a regular perturbation; one can easily check that $K_{0}=O\left(S^{-1}\right)$ as $S \rightarrow 0$. So, if we approximate $W$ and $P$ by

$$
W(\xi, \eta)=W_{0}(\xi, \eta)+\varepsilon W_{1}(\xi, \eta) \quad \text { and } \quad P(\xi, \eta)=P_{0}(\xi, \eta)+\varepsilon P_{1}(\xi, \eta)
$$

we find that $W_{0}$ solves

$$
H W_{0}=P_{0}
$$

and then $W_{1}$ solves

$$
H W_{1}=P_{1}-K_{0} W_{0},
$$

where $H$ is defined below (4.6) and

$$
\left(K_{0} W_{0}\right)\left(\xi_{0}, \eta_{0}\right)=\frac{1}{4 \pi} \int_{D} K_{0}\left(\zeta, \zeta_{0}\right) W_{0}(\xi, \eta) d \xi d \eta
$$

Both (5.3) and (5.4) are integral equations over the unit disc; they can be solved using the methods of Sec. 3. Then, using $\left|f^{\prime}(\zeta)\right| \simeq 1+\varepsilon \operatorname{Re}\left\{g^{\prime}(\zeta)\right\}$ for small $\varepsilon$, we obtain

$$
w(x(\zeta), y(\zeta))=a W_{0}+a \varepsilon\left(W_{1}-\frac{1}{2} W_{0} \operatorname{Re}\left\{g^{\prime}(\zeta)\right\}\right)
$$


as our approximation to the crack-opening displacement $w$, correct to first order in $\varepsilon$. Note that, in principle, the method can be extended to yield higher-order approximations.

In order to proceed, we must be able to calculate $K_{0} W_{0}$. Let us assume that $g$ is analytic in a domain that includes $D$, so that we can surround $D$ by a simple closed contour $C$. Then, using the residue theorem, we obtain

$$
K_{0}\left(\zeta, \zeta_{0}\right)=\frac{3}{2 S^{3}} \operatorname{Re}\left\{\frac{1}{2 \pi i} \int_{C} \frac{\left(\zeta-\zeta_{0}\right)^{2} g(\omega)}{(\omega-\zeta)^{2}\left(\omega-\zeta_{0}\right)^{2}} d \omega\right\},
$$

which is an integral representation for $K_{0}\left(\zeta, \zeta_{0}\right)$, involving the values of $g$ on $C$. Hence,

$$
\left(K_{0} W_{0}\right)\left(\zeta_{0}\right)=\frac{3}{2} \operatorname{Re}\left\{\frac{1}{2 \pi i} \int_{C} \frac{g(\omega)}{\left(\omega-\zeta_{0}\right)^{2}} L\left(\omega, \zeta_{0}\right) d \omega\right\}
$$

where

$$
L\left(\omega, \zeta_{0}\right)=H\left\{\frac{\left(\zeta-\zeta_{0}\right)^{2}}{(\omega-\zeta)^{2}} W_{0}(\zeta)\right\}
$$

is independent of the mapping $g$.

5.1. Uniform pressure. Suppose that the loading is uniform, so that $p=-\sigma$, a constant (recall the definition (2.1)). Hence,

$$
P_{0}=-\sigma \quad \text { and } \quad P_{1}=-\frac{3}{2} \sigma \operatorname{Re}\left\{g^{\prime}\left(\zeta_{0}\right)\right\}
$$

From Sec. 3, we easily obtain

$$
W_{0}=H^{-1} P_{0}=4(\sigma / \pi) \sqrt{1-s^{2}}
$$

Next, we evaluate $L$, defined by (5.7). We have

$$
\frac{\left(\zeta-\zeta_{0}\right)^{2}}{(\omega-\zeta)^{2}}=\sum_{m=0}^{\infty} \frac{(m+1)}{\omega^{m+2}}\left(\zeta^{m+2}-2 \zeta^{m+1} \zeta_{0}+\zeta^{m} \zeta_{0}^{2}\right)
$$

for $|\zeta / \omega|<1$ (which is satisfied since $|\zeta|<1$ and $|\omega|>1$ ). But $H\left\{\zeta^{n} \sqrt{1-s^{2}}\right\}$ can be evaluated using (3.3) and (3.4); hence,

$$
L\left(\omega, \zeta_{0}\right)=-4(\sigma / \pi) \sum_{m=0}^{\infty}(m+1) Z_{0}^{m+2} \Gamma(3 / 2) l_{m}
$$

where $Z_{0}=\zeta_{0} / \omega$ and

$$
l_{m}=\frac{\Gamma(m+7 / 2)}{(m+2) !}-2 \frac{\Gamma(m+5 / 2)}{(m+1) !}+\frac{\Gamma(m+3 / 2)}{m !}=-\frac{\Gamma(m+3 / 2)}{4(m+2) !} .
$$

The sum (5.8) can be evaluated; we have

$$
\begin{aligned}
L\left(\omega, \zeta_{0}\right) & =\frac{1}{2}(\sigma / \pi) Z_{0}^{2}\{\Gamma(3 / 2)\}^{2} F\left(2,3 / 2 ; 3 ; Z_{0}\right) \\
& =\frac{1}{2} \sigma\left\{\left(1-Z_{0}\right)^{-1 / 2}+\left(1-Z_{0}\right)^{1 / 2}-2\right\},
\end{aligned}
$$


where $F(a, b ; c ; z)$ is the Gauss hypergeometric function and we have used [1, Eq. 15.1.14].

Next, consider the right-hand side of (5.4). We have expressed $K_{0} W_{0}$ as a contour integral around $C$; so it is convenient to do the same for $P_{1}$ :

$$
P_{1}\left(\zeta_{0}\right)=-\frac{3 \sigma}{2} \operatorname{Re}\left\{\frac{1}{2 \pi i} \int_{C} \frac{g(\omega)}{\left(\omega-\zeta_{0}\right)^{2}} d \omega\right\} .
$$

Hence

$$
P_{1}-K_{0} W_{0}=-\frac{3 \sigma}{4} \operatorname{Re}\left\{\frac{1}{2 \pi i} \int_{C} \frac{g(\omega)}{\omega^{2}} Q\left(Z_{0}\right) d \omega\right\}
$$

where

$$
Q\left(Z_{0}\right)=\left(1-Z_{0}\right)^{-2}\left\{\left(1-Z_{0}\right)^{-1 / 2}+\left(1-Z_{0}\right)^{1 / 2}\right\} .
$$

So, if we expand in powers of $Z_{0}$, we can then apply $H^{-1}$, using (3.3) and (3.4) again. We have

$$
Q\left(Z_{0}\right)=\frac{2}{3} \sum_{m=0}^{\infty} \frac{(m+3) \Gamma(m+3 / 2)}{m ! \Gamma(3 / 2)} Z_{0}^{m}
$$

for $\left|Z_{0}\right|<1$, using

$$
\left(1-Z_{0}\right)^{-\alpha}=\sum_{m=0}^{\infty} \frac{\Gamma(m+\alpha)}{\Gamma(\alpha)} \frac{Z_{0}^{m}}{m !}
$$

Hence,

$$
H^{-1} Q=-\frac{8}{3 \pi} \sqrt{1-s^{2}} \sum_{m=0}^{\infty}(m+3)\left(\frac{\zeta}{\omega}\right)^{m},
$$

which is a combination of a geometric series and its derivative:

$$
H^{-1} Q=-\frac{8}{3 \pi} \sqrt{1-s^{2}}\left\{\frac{\omega^{2}}{(\omega-\zeta)^{2}}+\frac{2 \omega}{\omega-\zeta}\right\} .
$$

Next, we obtain $W_{1}$ from (5.4) by combining (5.9) and (5.10):

$$
\begin{aligned}
W_{1} & =H^{-1}\left(P_{1}-K_{0} W_{0}\right) \\
& =\frac{2 \sigma}{\pi} \sqrt{1-s^{2}} \operatorname{Re}\left\{\frac{1}{2 \pi i} \int_{C} \frac{g(\omega)}{\omega^{2}}\left(\frac{\omega^{2}}{(\omega-\zeta)^{2}}+\frac{2 \omega}{\omega-\zeta}\right) d \omega\right\} \\
& =\frac{2 \sigma}{\pi} \sqrt{1-s^{2}} \operatorname{Re}\left\{2 \frac{g(\zeta)}{\zeta}+g^{\prime}(\zeta)\right\},
\end{aligned}
$$

after using the residue theorem to evaluate the contour integral; note that the singularity at $\omega=0$ is removable, due to (5.2).

Finally, we substitute $W_{0}$ and $W_{1}$ into (5.5) to give

$$
w(x(\zeta), y(\zeta))=\frac{4 a \sigma}{\pi} \sqrt{1-s^{2}}\left\{1+\varepsilon \operatorname{Re}\left[\frac{g(\zeta)}{\zeta}\right]\right\} .
$$

This is our approximation for the crack-opening displacement; it is correct to first order in $\varepsilon$. 
5.2. Comparison with Gao and Rice. Let us express (5.11) in terms of the original variables. From (4.3) and (5.1), with $z=r e^{i \theta}$ and $\zeta=s e^{i \varphi}$, we have

$$
r=a s e^{i(\varphi-\theta)}+a \varepsilon e^{-i \theta} g(\zeta) .
$$

The imaginary part of this equation gives $\theta=\varphi+O(\varepsilon)$, whence the real part gives

$$
r=a s(1+\varepsilon G(s, \varphi)) \text {, }
$$

where

$$
G(s, \varphi)=\operatorname{Re}\left\{\zeta^{-1} g(\zeta)\right\}
$$

In particular, $\partial \Omega$ corresponds to $s=1$ :

$$
\rho(\theta)=a(1+\varepsilon G(\varphi))
$$

where

$$
G(\varphi)=G(1, \varphi)=\operatorname{Re}\left\{e^{-i \varphi} g\left(e^{i \varphi}\right)\right\}
$$

Hence, discarding terms of $O\left(\varepsilon^{2}\right)$,

$$
\rho^{2}-r^{2}=a^{2}\left(1-s^{2}\right)\left\{1+2 \varepsilon\left(1-s^{2}\right)^{-1}\left[G(\varphi)-s^{2} G(s, \varphi)\right]\right\}
$$

and so

$$
a \sqrt{1-s^{2}}=\sqrt{\rho^{2}-r^{2}}\left\{1-\varepsilon\left(1-s^{2}\right)^{-1}\left[G(\varphi)-s^{2} G(s, \varphi)\right]\right\} .
$$

Then, eliminating $\sqrt{1-s^{2}}$ from (5.11), we obtain

$$
w(x, y)=4(\sigma / \pi) \sqrt{\rho^{2}-r^{2}}\left\{1-\varepsilon\left(1-s^{2}\right)^{-1}[G(\varphi)-G(s, \varphi)]\right\} .
$$

Now, since $G(s, \varphi)$ is the real part of an analytic function, it is harmonic. Moreover, since it is harmonic in the unit disc, $0 \leq s<1$, it can be represented in terms of its boundary values on $s=1$ (namely, $G(\varphi)$ ) using Poisson's formula [2, p. 166]:

$$
G(s, \varphi)=\frac{1-s^{2}}{2 \pi} \int_{0}^{2 \pi} \frac{G\left(\varphi^{\prime}\right) d \varphi^{\prime}}{1+s^{2}-2 s \cos \left(\varphi-\varphi^{\prime}\right)}
$$

Also, since any constant is harmonic,

$$
G(\varphi)=\frac{1-s^{2}}{2 \pi} \int_{0}^{2 \pi} \frac{G(\varphi) d \varphi^{\prime}}{1+s^{2}-2 s \cos \left(\varphi-\varphi^{\prime}\right)}
$$

Consequently, (5.12) becomes

$$
w(x, y)=\frac{4 \sigma}{\pi} \sqrt{\rho^{2}-r^{2}}\left\{1+\frac{\varepsilon}{2 \pi} \int_{0}^{2 \pi} \frac{G\left(\varphi^{\prime}\right)-G(\varphi)}{1+s^{2}-2 s \cos \left(\varphi-\varphi^{\prime}\right)} d \varphi^{\prime}\right\} .
$$

In the integrand, we can use $a \varepsilon\left\{G\left(\varphi^{\prime}\right)-G(\varphi)\right\}=\rho\left(\theta^{\prime}\right)-\rho(\theta)$. Also, $\varphi=\theta, \varphi^{\prime}=\theta^{\prime}$, $a s=r$, and $a=\rho$, to leading order; so, finally, we obtain

$$
w(x, y)=\frac{4 \sigma}{\pi} \sqrt{[\rho(\theta)]^{2}-r^{2}}\left\{1+\frac{1}{2 \pi} \int_{0}^{2 \pi} \frac{\rho(\theta)\left[\rho\left(\theta^{\prime}\right)-\rho(\theta)\right]}{[\rho(\theta)]^{2}+r^{2}-2 r \rho(\theta) \cos \left(\theta-\theta^{\prime}\right)} d \theta^{\prime}\right\} .
$$

This formula expresses the new crack-opening displacement in terms of the geometry of the crack's edge. It agrees with a formula obtained previously by Gao and Rice [9, Eq. $\left(18^{\prime}\right)$ ], using an entirely different method based on three-dimensional weight functions.

We remark that there is an extensive Russian literature on almost circular cracks. Panasyuk's work is described in [23, Chapter IX] and in [9, Appendix B]; for a review, see [24]. There is also an interesting heuristic method due to Fabrikant [7], ostensibly applicable to cracks of any shape. Applications to fracture mechanics are discussed by Rice [27]. 
6. Discussion. In Sec. 4, we showed how to transform the simplest hypersingular integral equation over a bounded, simply-connected region $\Omega$ into a similar equation over a circular region. The method requires an appropriate conformal mapping; such mappings are catalogued in [15]. In particular, the method could be applied to potential flow past a square plate.

The method will also generalise to other scalar problems, such as that of acoustic scattering by a sound-hard plate. Here, the free-space fundamental solution is $\exp \{i k R\} / R$, where $k$ is the wavenumber; the singular behaviour of this function is the same as for potential flow $(k=0)$, and so the regularization method of Sec. 4 is applicable. For somewhat circular plates, the method of Sec. 5 could then be used to study low-frequency diffraction problems.

The method will also generalise to vector problems, such as the shear loading of a flat crack; the required Fourier-Gegenbauer expansions for penny-shaped cracks have been developed by Krenk [17].

All of these extensions and applications of the basic methods described herein are currently being made.

Appendix A. Effect of the mapping (4.1). The original kernel has a singularity at $R=0$, where

$$
\begin{gathered}
R^{2}=r^{2}+r_{0}^{2}-2 r r_{0} \cos \left(\theta-\theta_{0}\right)=s^{2} \rho^{2}+s_{0}^{2} \rho_{0}^{2}-2 s s_{0} \rho \rho_{0} \cos \left(\varphi-\varphi_{0}\right), \\
x=r \cos \theta=s \rho \cos \varphi, \quad y=r \sin \theta=s \rho \sin \varphi \\
x_{0}=r_{0} \cos \theta_{0}=s_{0} \rho_{0} \cos \varphi_{0}, \quad y_{0}=r_{0} \sin \theta_{0}=s_{0} \rho_{0} \sin \varphi_{0},
\end{gathered}
$$

$\rho \equiv \rho(\varphi)$ and $\rho_{0} \equiv \rho\left(\varphi_{0}\right)$. Since $\rho(\varphi)>0$, the only zero of $R$ is at $(s, \varphi)=\left(s_{0}, \varphi_{0}\right)$. Let us fix $s_{0}$ and $\varphi_{0}$, and expand $R$ in a neighbourhood of the fixed point $\left(s_{0}, \varphi_{0}\right)$. Relative to this point, the point $(s, \varphi)$ has polar coordinates $S$ and $\Phi$, defined by

$$
s \cos \left(\varphi-\varphi_{0}\right)=s_{0}+S \cos \Phi \text { and } s \sin \left(\varphi-\varphi_{0}\right)=S \sin \Phi .
$$

We expand $R$ for small $S$. We have

$$
\begin{aligned}
R^{2} & =s_{0}^{2} \rho_{0}^{2}+\rho^{2}\left(s_{0}^{2}+S^{2}+2 s_{0} S \cos \Phi\right)-2 s_{0} \rho \rho_{0}\left(s_{0}+S \cos \Phi\right) \\
& =s_{0}^{2}\left(\rho-\rho_{0}\right)^{2}+2 s_{0} S \rho\left(\rho-\rho_{0}\right) \cos \Phi+\rho^{2} S^{2},
\end{aligned}
$$

exactly. Since $\left(\varphi-\varphi_{0}\right)$ is small, we have $\rho-\rho_{0} \simeq\left(\varphi-\varphi_{0}\right) \rho_{0}^{\prime} \simeq\left(S / s_{0}\right) \rho_{0}^{\prime} \sin \Phi$, where the error is $O\left(S^{2}\right)$ and $\rho_{0}^{\prime} \equiv \rho^{\prime}\left(\varphi_{0}\right)$. Hence

$$
R^{2} \simeq S^{2} \kappa\left(\Phi ; \varphi_{0}\right)
$$

where

$$
\kappa\left(\Phi ; \varphi_{0}\right)=\rho_{0}^{2} \sin ^{2} \Phi+\left(\rho_{0} \cos \Phi+\rho_{0}^{\prime} \sin \Phi\right)^{2}
$$

is positive. So, locally,

$$
\frac{1}{R^{3}} \simeq \frac{\sigma\left(\Phi ; \varphi_{0}\right)}{S^{3}}
$$

where $\sigma\left(\Phi ; \varphi_{0}\right)=\left[\kappa\left(\Phi ; \varphi_{0}\right)\right]^{-3 / 2}$; this shows that the (isotropic) singularity at $R=0$ has been mapped into a singularity at $S=0$ of the same order $\left(S^{-3}\right)$, but with a strength that depends on the angle of approach, $\Phi$. 
Appendix B. The kernel $K$ is weakly singular. The kernel $K$ is defined by (4.4); we expand it for small $S$. Write

$$
f_{1}=\left(\zeta-\zeta_{0}\right) \frac{f^{\prime \prime}\left(\zeta_{0}\right)}{f^{\prime}\left(\zeta_{0}\right)} \quad \text { and } \quad f_{2}=\frac{\left(\zeta-\zeta_{0}\right)^{2}}{2} \frac{f^{\prime \prime \prime}\left(\zeta_{0}\right)}{f^{\prime}\left(\zeta_{0}\right)}
$$

thus, $f_{1}=O(S)$ and $f_{2}=O\left(S^{2}\right)$ as $S \rightarrow 0$. Hence,

$$
\begin{aligned}
f^{\prime}(\zeta) & \simeq f^{\prime}\left(\zeta_{0}\right)\left\{1+f_{1}+f_{2}\right\} \\
f(\zeta)-f\left(\zeta_{0}\right) & \simeq\left(\zeta-\zeta_{0}\right) f^{\prime}\left(\zeta_{0}\right)\left\{1+\frac{1}{2} f_{1}+\frac{1}{3} f_{2}\right\}
\end{aligned}
$$

and

$$
K \simeq \frac{1}{S^{3}}\left\{\frac{\left|1+f_{1}+f_{2}\right|^{3 / 2}}{\left|1+\frac{1}{2} f_{1}+\frac{1}{3} f_{2}\right|^{3}}-1\right\} .
$$

Now, for constants $\alpha, \beta$, and $\gamma$, we have

$$
\left|1+\alpha f_{1}+\beta f_{2}\right|^{\gamma} \simeq 1+\alpha \gamma u_{1}+\frac{1}{2} \gamma\left\{(\gamma-1) \alpha^{2} u_{1}^{2}+\alpha^{2} v_{1}^{2}+2 \beta u_{2}\right\}
$$

with an error of $O\left(S^{3}\right)$, where $f_{1}=u_{1}+i v_{1}$ and $f_{2}=u_{2}+i v_{2}$. Hence

$$
K \simeq S^{-3}\left\{\frac{3}{8}\left(v_{1}^{2}-u_{1}^{2}\right)+\frac{1}{2} u_{2}\right\}
$$

which is $O\left(S^{-1}\right)$ as $S \rightarrow 0$.

\section{REFERENCES}

[1] M. Abramowitz and I. A. Stegun, eds., Handbook of Mathematical Functions, Dover, New York, 1965

[2] L. V. Ahlfors, Complex Analysis, 2nd edition, McGraw-Hill, New York, 1966

[3] H. F. Bueckner, Field singularities and related integral representations, in Mechanics of Fracture 1, Methods of Analysis and Solutions of Crack Problems (ed. G. C. Sih), Noordhoff, Leyden, 1973, pp. 239-314

[4] H. D. Bui, Application des potentiels élastiques à l'étude des fissures planes de forme arbitraire en milieu tridimensionnel, C. R. Acad. Sci. Paris, Série A 280, 1157-1160 (1975)

[5] A. Erdélyi, W. Magnus, F. Oberhettinger, and F. G. Tricomi, Higher Transcendental Functions, vol. 2, McGraw-Hill, New York, 1953

[6] V. J. Ervin and E. P. Stephan, Collocation with Chebyshev polynomials for a hypersingular integral equation on an interval, J. Comput. Appl. Math. 43, 221-229 (1992)

[7] V. I. Fabrikant, Flat crack of arbitrary shape in an elastic body: analytical approach, Philos. Mag. A. 56, 175-189 (1987)

[8] A. Frenkel, A Chebyshev expansion of singular integrodifferential equations with a $\partial^{2} \ln |s-t| / \partial s \partial t$ kernel, J. Comput. Phys. 51, 335-342 (1983)

[9] H. Gao and J. R. Rice, Somewhat circular tensile cracks, Internat. J. Fracture 33, 155-174 (1987)

[10] M. A. Golberg, The convergence of several algorithms for solving integral equations with finite-part integrals, J. Integral Equations 5, 329-340 (1983)

[11] M. A. Golberg, The convergence of several algorithms for solving integral equations with finite-part integrals. II, J. Integral Equations 9, 267-275 (1985)

[12] J. T. Guidera and R. W. Lardner, Penny-shaped cracks, J. Elasticity 5, 59-73 (1975)

[13] N. I. Ioakimidis, Application of finite-part integrals to the singular integral equations of crack problems in plane and three-dimensional elasticity, Acta Mech. 45, 31-47 (1982)

[14] A. C. Kaya and F. Erdogan, On the solution of integral equations with strongly singular kernels, Quart. Appl. Math. 45, 105-122 (1987) 
[15] H. Kober, Dictionary of Conformal Representations, Dover, New York, 1957

[16] E. Kossecka, Defects as surface distributions of double forces, Arch. Mech. 23, 481-494 (1971)

[17] S. Krenk, A circular crack under asymmetric loads and some related integral equations, J. Appl. Mech. 46, 821-826 (1979)

[18] G. Krishnasamy, L. W. Schmerr, T. J. Rudolphi, and F. J. Rizzo, Hypersingular boundary integral equations: some applications in acoustic and elastic wave scattering, J. Appl. Mech. 57, 404-414 (1990)

[10] P. A. Martin, The discontinuity in the elastostatic displacement vector across a penny-shaped crack under arbitrary loads, J. Elasticity 12, 201-218 (1982)

[20] P. A. Martin, Orthogonal polynomial solutions for pressurized elliptical cracks, Quart. J. Mech. Appl. Math. 39, 269-287 (1986)

[21] P. A. Martin, End-point behaviour of solutions to hypersingular integral equations, Proc. Roy. Soc. London Ser. A 432, 301-320 (1991)

[22] P. A. Martin and F. J. Rizzo, On boundary integral equations for crack problems, Proc. Roy. Soc. London Ser. A 421, 341-355 (1989)

[23] V. V. Panasyuk, Limiting Equilibrium of Brittle Solids with Fractures, Management Information Services, Detroit, 1970. [Lib. of Congr. No. 70-135093] (Translation from Russian edition, Naukova Dumka, Kiev, 1968)

[24] V. V. Panasyuk, A. E. Andrejkiv, and M. M. Stadnik, Three-dimensional static crack problems solution (a review), Engrg. Fract. Mech. 14, 245-260 (1981)

[25] N. F. Parsons and P. A. Martin, Scattering of water waves by submerged plates using hypersingular integral equations, Appl. Ocean Res. 14, 313-321 (1992)

[26] E. Z. Polch, T. A. Cruse, and C.-J. Huang, Traction BIE solutions for flat cracks, Computational Mech. 2, 253-267 (1987)

[27] J. R. Rice, Weight function theory for three-dimensional elastic crack analysis, in Fracture Mechanics: Perspectives and Directions (Twentieth Symposium) (ed. R. P. Wei and R. P. Gangloff), American Society for Testing and Materials, Philadelphia, 1989, pp. 29-57

[28] K. Takakuda, T. Koizumi, and T. Shibuya, On integral equation methods for crack problems, Bull. Japan. Soc. Mech. Engrs. 28, 217-224 (1985)

[29] J. Weaver, Three-dimensional crack analysis, Internat. J. Solids and Structures 13, 321-330 (1977) 